\title{
Quadratically Constrained Beamforming Robust Against Direction-of-Arrival Mismatch
}

\author{
Chun-Yang Chen, Student Member, IEEE, and P. P. Vaidyanathan, Fellow, IEEE
}

\begin{abstract}
It is well known that the performance of the minimum variance distortionless response (MVDR) beamformer is very sensitive to steering vector mismatch. Such mismatches can occur as a result of direction-of-arrival (DOA) errors, local scattering, near-far spatial signature mismatch, waveform distortion, source spreading, imperfectly calibrated arrays and distorted antenna shape. In this paper, an adaptive beamformer that is robust against the DOA mismatch is proposed. This method imposes two quadratic constraints such that the magnitude responses of two steering vectors exceed unity. Then, a diagonal loading method is used to force the magnitude responses at the arrival angles between these two steering vectors to exceed unity. Therefore, this method can always force the gains at a desired range of angles to exceed a constant level while suppressing the interferences and noise. A closed-form solution to the proposed minimization problem is introduced, and the diagonal loading factor can be computed systematically by a proposed algorithm. Numerical examples show that this method has excellent signal-to-interference-plus-noise ratio performance and a complexity comparable to the standard MVDR beamformer.
\end{abstract}

Index Terms-Capon beamformer, diagonal loading, direction-of-arrival (DOA) mismatch, minimum variance distortionless response (MVDR) beamformer, robust beamforming, steering vector uncertainty.

\section{INTRODUCTION}

B EAMFORMING has long been used in many areas, such as radar, sonar, seismology, medical imaging, speech processing, and wireless communications. An introduction to beamforming can be found in [25]-[30] and the references therein.

A data-dependent beamformer was proposed by Capon in [1]. By exploiting the second-order statistics of the array output, the method constrains the response of the signal of interest (SOI) to be unity and minimizes the variance of the beamformer output. This method is called minimum variance distortionless response (MVDR) beamformer in the literature. The MVDR beamformer has very good resolution, and the signal-to-interference-plus-noise ratio (SINR) performance is much better than traditional data-independent beamformers. However, when the steering vector of the SOI is imprecise, the response of the SOI is no longer constrained to be unity and is thus attenuated by the MVDR beamformer while minimizing

Manuscript received March 29, 2006; revised October 22, 2006. This work was supported in part by the ONR under Grant N00014-06-1-0011 and in part by The California Institute of Technology. The associate editor coordinating the review of this manuscript and approving it for publication was Dr. A. Rahim Leyman.

The authors are with the California Institute of Technology (Caltech), Pasadena, CA 91125 USA (e-mail: cyc@caltech.edu).

Digital Object Identifier 10.1109/TSP.2007.894402 the total variance of the beamformer output [2]. The effect is called signal cancellation. It dramatically degrades the output SINR. Many approaches, including [6]-[24] and the references therein, have been proposed for improving the robustness of the MVDR beamformer. A good introduction to this topic can be found in [3]. The steering vector of the SOI can be imprecise because of various reasons, such as direction-of-arrival (DOA) errors, local scattering, near-far spatial signature mismatch, waveform distortion, source spreading, imperfectly calibrated arrays, and distorted antenna shape [3], [4]. In this paper, we focus on DOA uncertainty.

There are many methods developed for solving the DOA mismatch problem. In [13]-[20], linear constraints have been imposed when minimizing the output variance. The linear constraints can be designed to broaden the main beam of the beampattern. These beamformers are called linearly constrained minimum variance (LCMV) beamformers. In [22] and [23], convex quadratic constraints have been used. In [21], a Bayesian approach has been used. For other types of mismatches, diagonal loading [11], [12] is known to provide robustness. However, the drawback of the diagonal loading method is that it is not clear how to choose a diagonal loading factor. In [24], the steering vector has been projected onto the signal-plus-interference subspace to reduce the mismatch. In [5], the magnitude responses of the steering vectors in a polyhedron set are constrained to exceed unity while the output variance is minimized. This method avoids the signal cancellation when the actual steering vector is in the designed polyhedron set. In [6], Vorobyov et al. have used a nonconvex constraint which forces the magnitude responses of the steering vectors in a sphere set to exceed unity. This nonconvex optimization problem has been reformulated in a convex form as a second-order cone programming (SOCP) problem. It has been also proven in [6] that this beamformer belongs to the family of diagonal loading beamformers. In [7] and [8], the sphere uncertainty set has been generalized to an ellipsoid set and the SOCP has been avoided by the proposed algorithms which efficiently calculate the corresponding diagonal loading level. In [9], a general rank case has been considered using a similar idea as in [6] and an elegant closed-form solution has been obtained.

In [5]-[9], the magnitude responses of steering vectors in an uncertainty set have been forced to exceed unity while minimizing the output variance. The uncertainty set has been selected as polyhedron, sphere, or ellipsoid in order to be robust against general types of steering vector mismatches. In this paper, we consider only the DOA mismatch. Inspired by these uncertainty-based methods, we consider a simplified uncertainty set which contains only the steering vectors with a 
desired uncertainty range of DOA. To find a suboptimal solution for this problem, the constraint is first loosened to two nonconvex quadratic constraints such that the magnitude responses of two steering vectors exceed unity. Then, a diagonal loading method is used to force the magnitude responses at the arrival angles between these two steering vectors to exceed unity. Therefore, this method can always force the gains at a desired range of angles to exceed a constant level while suppressing the interference and noise. A closed-form solution to the proposed minimization problem is introduced, and the diagonal loading factor can be computed systematically by a proposed iterative algorithm. Numerical examples show that this method has excellent SINR performance and a complexity comparable to the standard MVDR beamformer.

The rest of this paper is organized as follows: The MVDR beamformer and the analysis of steering vector mismatch are presented in Section II. Some previous work on robust beamforming is reviewed in Section III. In Section IV, we develop the theory and the algorithm of our new robust beamformer. Numerical examples are presented in Section V. Finally, conclusions are presented in Section VI.

Notations: Boldfaced lowercase letters such as y represent vectors, and boldfaced uppercase letters, such as $\mathbf{R}_{\mathbf{y}}$, denote matrices. The element in row $n$ and column $m$ of matrix $\mathbf{R}_{\mathbf{y}}$ is denoted by $R_{y, n, m}$. The notation $\mathbf{y}^{\dagger}$ denotes the conjugate transpose of the vector $\mathbf{y}$. Notation $E[x]$ denotes the expectation of the random variable $x$.

\section{MVDR BEAMFORMER AND THE STEERING VECTOR MISMATCH}

Consider a uniform linear array (ULA) of $N$ omnidirectional sensors with interelement spacing $d$. The SOI is a narrowband plane wave impinging from angle $\theta$. The baseband array output $\mathbf{y}(t)$ can be expressed as

$$
\mathbf{y}(t)=x(t) \mathbf{s}(\theta)+\mathbf{v}(t)
$$

where $\mathbf{v}(t)$ denotes the sum of the interferences and the noises, $x(t)$ is the $\mathbf{S O I}$, and $\mathbf{s}(\theta)$ represents the baseband array response of the SOI. It is called the "steering vector" and can be expressed as

$$
\mathbf{s}(\theta) \triangleq\left(\begin{array}{llll}
1 & \left.e^{j(2 \pi / \lambda) d \sin \theta} \quad \ldots \quad e^{j(N-1)(2 \pi / \lambda) d \sin \theta}\right)^{T}
\end{array}\right.
$$

where $\lambda$ is the operating wavelength. The output of the beamformer can be expressed as $\mathbf{w}^{\dagger} \mathbf{y}(t)$, where $\mathbf{w}$ is the complex weighting vector. The output signal-to-interferences-plus-noise ratio (SINR) of the beamformer is defined as

$$
\mathrm{SINR} \triangleq \frac{E\left|x(t) \mathbf{w}^{\dagger} \mathbf{s}(\theta)\right|^{2}}{E\left|\mathbf{w}^{\dagger} \mathbf{v}(t)\right|^{2}}=\frac{\sigma_{x}^{2}\left|\mathbf{w}^{\dagger} \mathbf{s}(\theta)\right|^{2}}{\mathbf{w}^{\dagger} \mathbf{R}_{\mathbf{v}} \mathbf{w}}
$$

where $\mathbf{R}_{\mathbf{v}} \triangleq E\left[\mathbf{v}(t) \mathbf{v}^{\dagger}(t)\right]$, and $\sigma_{x}^{2} \triangleq E\left[|x(t)|^{2}\right]$. By varying the weighting factors, the output SINR can be maximized by minimizing the total output variance while constraining the SOI response to be unity. This can be written as the following optimization problem:

$$
\begin{array}{cl}
\min _{\mathbf{w}} & \mathbf{w}^{\dagger} \mathbf{R}_{\mathbf{y}} \mathbf{w} \\
\text { subject to } & \mathbf{s}^{\dagger}(\theta) \mathbf{w}=1
\end{array}
$$

where $\mathbf{R}_{\mathbf{y}} \triangleq E\left[\mathbf{y}(t) \mathbf{y}^{\dagger}(t)\right]$. This is equivalent to minimizing $\mathbf{w}^{\dagger} \mathbf{R}_{\mathbf{v}} \mathbf{w}$ subject to $\left|\mathbf{s}^{\dagger}(\theta) \mathbf{w}\right|=1$ because

$$
\begin{aligned}
\mathbf{w}^{\dagger} \mathbf{R}_{\mathbf{y}} \mathbf{w} & =\mathbf{w}^{\dagger} \mathbf{R}_{\mathbf{v}} \mathbf{w}+\sigma_{x}^{2}\left|\mathbf{s}^{\dagger}(\theta) \mathbf{w}\right|^{2} \\
& =\mathbf{w}^{\dagger} \mathbf{R}_{\mathbf{v}} \mathbf{w}+\sigma_{x}^{2} \cdot 1 .
\end{aligned}
$$

The solution to this problem is well known and was first given by Capon in [1] as

$$
\mathbf{w}_{c}=\frac{\mathbf{R}_{\mathbf{y}}{ }^{-1} \mathbf{s}(\theta)}{\mathbf{s}^{\dagger}(\theta) \mathbf{R}_{\mathbf{y}}{ }^{-1} \mathbf{s}(\theta)} .
$$

This beamformer is called the MVDR beamformer in the literature. When there is a mismatch between the actual arrival angle $\theta$ and the assumed arrival angle $\theta_{m}$, this beamformer becomes

$$
\mathbf{w}_{m}=\frac{\mathbf{R}_{\mathbf{y}}{ }^{-1} \mathbf{s}\left(\theta_{m}\right)}{\mathbf{s}^{\dagger}\left(\theta_{m}\right) \mathbf{R}_{\mathbf{y}}{ }^{-1} \mathbf{s}\left(\theta_{m}\right)} .
$$

It can be viewed as the solution to the minimization problem

$$
\begin{array}{cl}
\min _{\mathbf{w}} & \mathbf{w}^{\dagger} \mathbf{R}_{\mathbf{y}} \mathbf{w} \\
\text { subject to } & \mathbf{s}^{\dagger}\left(\theta_{m}\right) \mathbf{w}=1 .
\end{array}
$$

Since $\mathbf{w}^{\dagger} \mathbf{R}_{\mathbf{y}} \mathbf{w}=\mathbf{w}^{\dagger} \mathbf{R}_{\mathbf{v}} \mathbf{w}+\sigma_{x}^{2}\left|\mathbf{s}^{\dagger}(\theta) \mathbf{w}\right|^{2}$, and $\mathbf{s}^{\dagger}(\theta) \mathbf{w}=$ 1 is no longer valid due to the mismatch, the SOI magnitude response might be attenuated as a part of the objective function. This suppression leads to severe degradation in SINR, because the SOI is treated as interference in this case. The phenomenon is called "signal cancellation." A small mismatch can lead to severe degradation in the SINR.

\section{Previous Work ON Robust BeAmForming}

Many approaches have been proposed for improving the robustness of the standard MVDR beamformer. In this section, we briefly mention some of them related to our work.

\section{A. Diagonal Loading Method}

In [11] and [12], the optimization problem in (3) is modified as

$$
\begin{aligned}
\min _{\mathbf{w}} & \mathbf{w}^{\dagger}\left(\mathbf{R}_{\mathbf{y}}+\gamma \mathbf{I}_{\mathbf{N}}\right) \mathbf{w} \\
\text { subject to } & \mathbf{s}^{\dagger}(\theta) \mathbf{w}=1 .
\end{aligned}
$$

This approach is called diagonal loading in the literature. It increases the variance of the artificial white noise by the amount $\gamma$. This modification forces the beamformer to put more effort in suppressing white noise rather than interference. As before, 
when the SOI steering vector is mismatched, the SOI is attenuated as one type of interference. As the beamformer puts less effort in suppressing the interferences and noise, the signal cancellation problem addressed in Section II is reduced. However, when $\gamma$ is too large, the beamformer fails to suppress strong interference because it puts most effort to suppress the white noise. Hence, there is a tradeoff between reducing signal cancellation and effectively suppressing interference. For that reason, it is not clear how to choose a good diagonal loading factor $\gamma$ in the traditional MVDR beamformer.

\section{B. LCMV Method}

In [13]-[20], the linear constraint of the MVDR in (3) has been generalized to a set of linear constraints as

$$
\begin{aligned}
\min _{\mathbf{w}} & \mathbf{w}^{\dagger} \mathbf{R}_{\mathbf{y}} \mathbf{w} \\
\text { subject to } & \mathbf{C}^{\dagger} \mathbf{w}=\mathbf{f}
\end{aligned}
$$

where $\mathbf{C}^{\dagger}$ is an $L \times N$ matrix and $\mathbf{f}$ is an $L \times 1$ vector. The solution can be found by using the Lagrange multiplication method as

$$
\mathbf{w}_{\mathbf{l}}=\mathbf{R}_{\mathbf{y}}{ }^{-1} \mathbf{C}\left(\mathbf{C}^{\dagger} \mathbf{R}_{\mathbf{y}}{ }^{-1} \mathbf{C}\right)^{-1} f .
$$

This is called the LCMV beamformer. These linear constraints can be directional constraints [15], [16] or derivative constraints [17]-[19]. The directional constraints force the responses of multiple neighbor steering vectors to be unity. The derivative constraints not only force the response to be unity but also several orders of the derivatives of the beampattern in the assumed DOA to be zero. These constraints broaden the main beam of the beampattern so that it is more robust against the DOA mismatch. In [20], linear constraints have further been used to allow an arbitrary specification of the quiescent response.

\section{Extended Diagonal Loading Method}

In [6], the following optimization problem is considered:

$$
\begin{array}{cl}
\min _{\mathbf{w}} & \mathbf{w}^{\dagger} \mathbf{R}_{\mathbf{y}} \mathbf{w} \\
\text { subject to } & \left|\mathbf{w}^{\dagger} \mathbf{s}\right| \geq 1 \quad \forall \mathbf{s} \in \mathcal{E}
\end{array}
$$

where $\mathcal{E}$ is a sphere defined as

$$
\mathcal{E}=\{\overline{\mathbf{s}}+\mathbf{e}\|\mathbf{e}\| \leq \epsilon\}
$$

where $\overline{\mathbf{s}}$ is the assumed steering vector. The constraint forces the magnitude responses of an uncertainty set of steering vectors to exceed unity. The constraint is actually nonconvex. However, in [6], it is reformulated to a second-order cone programming (SOCP) problem which can be solved by using some existing tools, such as SeDuMi in MATLAB. It has also been proven in [6] that the solution to (8) has the form $c\left(\mathbf{R}_{\mathbf{y}}+\gamma \mathbf{I}_{\mathbf{N}}\right)^{-1} \overline{\mathbf{s}}$ for some appropriate $c$ and $\gamma$. Therefore, this method can be viewed as an extended diagonal loading method [7]. In [7] and [8], the uncertainty set in (9) has been generalized to an ellipsoid, and the SOCP has been avoided by the proposed algorithms which directly calculate the corresponding diagonal loading level $\gamma$ as a function of $\mathbf{R}_{\mathbf{y}}, \overline{\mathbf{s}}$, and $\epsilon$.

\section{General-Rank Method}

In [9], a general-rank signal model is considered. The steering vector $\mathbf{s}$ is assumed to be a random vector that has a covariance $\mathbf{R}_{\mathrm{s}}$. The mismatch is therefore modeled as an error matrix $\boldsymbol{\Delta}_{1} \in \mathbf{C}^{N \times N}$, in the signal covariance matrix $\mathbf{R}_{\mathbf{s}}$, and an error matrix $\Delta_{2} \in \mathbf{C}^{N \times N}$ in the output covariance matrix $\mathbf{R}_{\mathbf{y}}$. The following optimization problem is considered:

$$
\begin{aligned}
& \min _{\mathbf{w}} \max _{\left\|\boldsymbol{\Delta}_{2}\right\|_{F} \leq \gamma} \mathbf{w}^{\dagger}\left(\mathbf{R}_{\mathbf{y}}+\boldsymbol{\Delta}_{2}\right) \mathbf{w} \\
& \text { subject to } \mathbf{w}^{\dagger}\left(\mathbf{R}_{\mathbf{s}}+\boldsymbol{\Delta}_{1}\right) \mathbf{w} \geq 1 \forall\left\|\boldsymbol{\Delta}_{1}\right\|_{F} \leq \epsilon
\end{aligned}
$$

where $\|\Delta\|_{F}$ denotes the Frobenius norm of the matrix $\Delta$, and $\epsilon$ and $\gamma$ are the upperbounds of the Frobenius norms of the error matrices $\Delta_{1}$ and $\Delta_{2}$, respectively. This optimization problem has an elegant closed-form solution as shown by Shahbazpanahi et al. in [9], namely

$$
\mathbf{w}_{\mathbf{n}}=P\left\{\left(\mathbf{R}_{\mathbf{y}}+\gamma \mathbf{I}_{N}\right)^{-1}\left(\mathbf{R}_{\mathbf{s}}-\epsilon \mathbf{I}_{N}\right)\right\}
$$

where $P\{\mathbf{A}\}$ denotes the principal eigenvector of the matrix A. The principal eigenvector is defined as the eigenvector corresponding to the largest eigenvalue.

\section{NEW ROBUST BEAMFORMER}

In this paper, we consider the DOA mismatch. When there is a mismatch, the minimization in (6) suppresses the magnitude response of the SOI. To avoid this, we should force the magnitude responses at a range of arrival angles to exceed unity while minimizing the total output variance. This optimal robust beamformer problem can be expressed as

$$
\begin{aligned}
\mathbf{w}_{d}= & \arg \min _{\mathbf{w}} \mathbf{w}^{\dagger} \mathbf{R}_{\mathbf{y}} \mathbf{w} \\
& \text { subject to }\left|\mathbf{s}^{\dagger}(\theta) \mathbf{w}\right|^{2} \geq 1 \text { for } \theta_{1} \leq \theta \leq \theta_{2}
\end{aligned}
$$

where $\theta_{1}$ and $\theta_{2}$ are the lower and upperbounds of the uncertainty of SOI arrival angle, respectively, and $\mathbf{s}(\theta)$ is the steering vector defined in (1) with the arrival angle $\theta$. The following uncertainty set of steering vectors is considered:

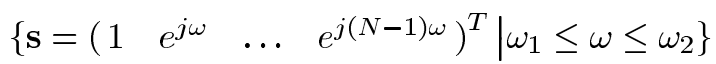

where $\omega_{1} \triangleq 2 \pi \sin \theta_{1} / \lambda$, and $\omega_{2} \triangleq 2 \pi \sin \theta_{2} / \lambda$. This uncertainty set is a curve. This constraint protects the signals in the range of angles $\theta_{1} \leq \theta \leq \theta_{2}$ from being suppressed.

\section{A. Frequency-Domain View of the Problem}

Substituting (12) into the constraint in (11), the constraint can be rewritten as

$$
\left|\sum_{n=0}^{N-1} w_{n} e^{-j \omega n}\right|=\left|W\left(e^{j \omega}\right)\right| \geq 1 \text { for } \omega_{1} \leq \omega \leq \omega_{2}
$$



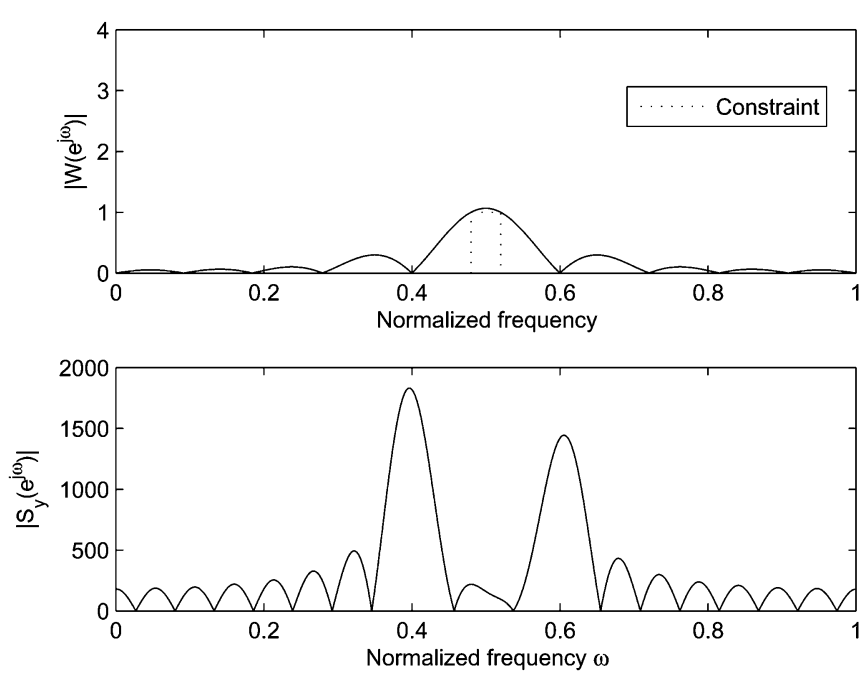

Fig. 1. Frequency-domain view of the optimization problem.

where $W\left(e^{j \omega}\right)$ is the Fourier transform of the weight vector $\mathbf{w}$. The objective function $\mathbf{w}^{\dagger} \mathbf{R}_{\mathbf{y}} \mathbf{w}$ can also be rewritten in the frequency domain as

$$
\begin{aligned}
\mathbf{w}^{\dagger} \mathbf{R}_{\mathbf{y}} \mathbf{w} & =\sum_{n=0}^{N-1} \sum_{m=0}^{N-1} w_{n}^{*} R_{y, n, m} w_{m} \\
& =\sum_{n=0}^{N-1} \sum_{m=0}^{N-1} w_{n}^{*} r_{y}(n-m) w_{m} \\
& =\frac{1}{2 \pi} \int_{0}^{2 \pi}\left|W\left(e^{j \omega}\right)\right|^{2} S_{y}\left(e^{j \omega}\right) d \omega
\end{aligned}
$$

where $S_{y}\left(e^{j \omega}\right)$ is the power spectral density (PSD) of the array output $\mathbf{y}$. Therefore, the optimization problem can be rewritten in the frequency domain as

$$
\begin{aligned}
& \min _{\mathbf{w}} \int_{0}^{2 \pi}\left|W\left(e^{j \omega}\right)\right|^{2} S_{y}\left(e^{j \omega}\right) d \omega \\
& \text { subject to }\left|W\left(e^{j \omega}\right)\right| \geq 1 \text { for } \omega_{1} \leq \omega \leq \omega_{2} .
\end{aligned}
$$

Note that $S_{y}\left(e^{j \omega}\right)$ is a weighting function in the above integral. The frequency-domain view of this optimization problem is illustrated in Fig. 1. The integral of $\left|W\left(e^{j \omega}\right)\right|^{2} S_{y}\left(e^{j w}\right)$ is minimized while $\left|W\left(e^{j \omega}\right)\right| \geq 1$ for $\omega_{1} \leq \omega \leq \omega_{2}$ is satisfied. Even though we will not solve the problem in the frequency domain, it is insightful to look at it this way.

\section{B. Two-Point Quadratic Constraint}

It is not clear how to solve the optimal beamformer $\mathbf{w}_{d}$ in (11) because the constraint does not fit into any of the existing standard optimization methods. The constraint $\left|\mathbf{s}^{\dagger}(\theta) \mathbf{w}\right|^{2} \geq 1$ for $\theta_{1} \leq \theta \leq \theta_{2}$ can be viewed as an infinite number of nonconvex quadratic constraints. To find a suboptimal solution, we start looking for the solution by loosening the constraint. We first loosen the constraint by choosing only two constraints $\left|\mathbf{s}^{\dagger}\left(\theta_{1}\right) \mathbf{w}\right|^{2} \geq 1$ and $\left|\mathbf{s}^{\dagger}\left(\theta_{2}\right) \mathbf{w}\right|^{2} \geq 1$ from the infinite con- straints $\left|\mathbf{s}^{\dagger}(\theta) \mathbf{w}\right|^{2} \geq 1$ for $\theta_{1} \leq \theta \leq \theta_{2}$. The corresponding optimization problem can be written as

$$
\begin{aligned}
\min _{\mathbf{w}} & \mathbf{w}^{\dagger} \mathbf{R}_{\mathbf{y}} \mathbf{w} \\
\text { subject to } & \left|\mathbf{s}^{\dagger}\left(\theta_{1}\right) \mathbf{w}\right|^{2} \geq 1 \text { and }\left|\mathbf{s}^{\dagger}\left(\theta_{2}\right) \mathbf{w}\right|^{2} \geq 1 .
\end{aligned}
$$

Due to the fact that the constraint is loosened, the minimum to this problem is a lowerbound of the original problem in (11). Note that the constraint in (13) is a nonconvex quadratic constraint. In order to obtain an analytic solution, we reformulate the problem in the following equivalent form:

$$
\begin{aligned}
\min _{\mathbf{w}, \phi, \rho_{0} \geq 1, \rho_{1} \geq 1} & \mathbf{w}^{\dagger} \mathbf{R}_{\mathbf{y}} \mathbf{w} \\
\text { subject to } & \mathbf{S}^{\dagger} \mathbf{w}=\left(\begin{array}{c}
\rho_{0} \\
\rho_{1} e^{j \phi}
\end{array}\right),
\end{aligned}
$$

where

$$
\mathbf{S}=\left(\begin{array}{ll}
\mathbf{s}\left(\theta_{1}\right) & \mathbf{s}\left(\theta_{2}\right)
\end{array}\right)
$$

and $\rho_{0}, \rho_{1}$, and $\phi$ are real numbers.

To solve this problem, we divide it into two parts. We first assume $\phi, \rho_{0}$, and $\rho_{1}$ are constants and solve $\mathbf{w}$. The solution $\mathbf{w}$ will be a function of $\phi, \rho_{0}$, and $\rho_{1}$. Then, the solution $\mathbf{w}$ can be substituted back into the objective function so that the objective function becomes a function of $\phi, \rho_{0}$, and $\rho_{1}$. Finally, we minimize the new objective function by choosing $\phi, \rho_{0}$, and $\rho_{1}$. Define the function

$$
L(\mathbf{w}, \mathbf{b})=\mathbf{w}^{\dagger} \mathbf{R}_{\mathbf{y}} \mathbf{w}-\mathbf{b}^{\dagger} \mathbf{S}^{\dagger} \mathbf{w}
$$

where $\mathbf{b} \in \mathbf{C}^{2}$ is the Lagrange multiplier. Taking the gradient of (14) and equating it to zero, we obtain the solution

$$
\mathbf{w}_{0}=\mathbf{R}_{\mathbf{y}}^{-1} \mathbf{S b}
$$

Substituting the above equation into the constraint, the Lagrange multiplier can be expressed as

$$
\mathbf{b}=\left(\mathbf{S}^{\dagger} \mathbf{R}_{\mathbf{y}}{ }^{-1} \mathbf{S}\right)^{-1}\left(\begin{array}{c}
\rho_{0} \\
\rho_{1} e^{j \phi}
\end{array}\right) .
$$

Substituting $\mathbf{b}$ back into $\mathbf{w}_{0}$, we obtain

$$
\mathbf{w}_{0}=\mathbf{R}_{\mathbf{y}}{ }^{-1} \mathbf{S}\left(\mathbf{S}^{\dagger} \mathbf{R}_{\mathbf{y}}{ }^{-1} \mathbf{S}\right)^{-1}\left(\begin{array}{c}
\rho_{0} \\
\rho_{1} e^{j \phi}
\end{array}\right) .
$$

Given $\phi, \rho_{0}$, and $\rho_{1}, \mathbf{w}_{0}$ can be found from the above equation. Note that it is exactly the solution to the LCMV beamformer mentioned in Section III-B with two directional constraints. Therefore, this approach can be viewed as an LCMV beamformer with a further optimized $\mathbf{f}$ in (7). However, this approach is reformulated from the nonconvex quadratic problem in (13). It is intrinsically different from a linearly constrained problem. The task now is to solve for $\phi, \rho_{0}$, and $\rho_{1}$. Write

$$
\left(\mathbf{S}^{\dagger} \mathbf{R}_{\mathbf{y}}{ }^{-1} \mathbf{S}\right)^{-1}=\left(\begin{array}{cc}
r_{0} & r_{2} e^{j \beta} \\
r_{2} e^{-j \beta} & r_{1}
\end{array}\right)
$$


where $r_{0}, r_{1}$, and $r_{2}$ are real non-negative numbers. Substituting $\mathbf{w}_{0}$ in (15) into the objective function, it becomes

$$
\begin{aligned}
\mathbf{w}_{0}^{\dagger} \mathbf{R}_{\mathbf{y}} \mathbf{w}_{0} & =\left(\begin{array}{ll}
\rho_{0} & \rho_{1} e^{-j \phi}
\end{array}\right)\left(\mathbf{S}^{\dagger} \mathbf{R}_{\mathbf{y}}{ }^{-1} \mathbf{S}\right)^{-1}\left(\begin{array}{c}
\rho_{0} \\
\rho_{1} e^{j \phi}
\end{array}\right) \\
& =r_{0} \rho_{0}^{2}+r_{1} \rho_{1}^{2}+2 \operatorname{Re}\left\{r_{2} \rho_{0} \rho_{1} e^{j(\beta+\phi)}\right\} \\
& \geq r_{0} \rho_{0}^{2}+r_{1} \rho_{1}^{2}-2 r_{2} \rho_{0} \rho_{1} .
\end{aligned}
$$

To minimize the objective function, $\phi$ can be chosen as

$$
\phi=-\beta+\pi
$$

so that the last equality in (16) holds. Now $\phi$ and $\mathbf{w}_{0}$ are obtained by (17) and (15), and the objective function becomes (16). To further minimize the objective function, $\rho_{0}$ and $\rho_{1}$ can be found by solving the following optimization problem:

$$
\min _{\rho_{0} \geq 1, \rho_{1} \geq 1} r_{0} \rho_{0}^{2}+r_{1} \rho_{1}^{2}-2 r_{2} \rho_{0} \rho_{1}
$$

This can be solved by using the Karush-Kuhn-Tucker (KKT) condition. The following solution can be obtained:

$$
\begin{aligned}
& \rho_{0}= \begin{cases}1, & \frac{r_{2}}{r_{0}} \leq 1 \\
\frac{r_{2}}{r_{0}}, & \frac{r_{2}}{r_{0}}>1,\end{cases} \\
& \rho_{1}= \begin{cases}1, & \frac{r_{2}}{r_{1}} \leq 1 \\
\frac{r_{2}}{r_{1}}, & \frac{r_{2}}{r_{1}}>1 .\end{cases}
\end{aligned}
$$

Summarizing (15), (17), and (18), the following algorithm for solving the beamformer with the two-point quadratic constraint in (13) is obtained.

\section{Algorithm 1}

Given $\theta_{1}, \theta_{2}$, and $\mathbf{R}_{\mathbf{y}}$, compute $\mathbf{w}_{0}$ by the following steps:

1. $\mathbf{S} \leftarrow\left(\begin{array}{ll}\mathbf{s}\left(\theta_{1}\right) & \left.\mathbf{s}\left(\theta_{2}\right)\right) .\end{array}\right.$

2. $\mathbf{V} \leftarrow\left(\mathbf{R}_{\mathbf{y}}\right)^{-1} \mathbf{S}$.

3. $\mathbf{R} \triangleq\left(\begin{array}{cc}r_{0} & r_{2} e^{j \beta} \\ r_{2} e^{-j \beta} & r_{1}\end{array}\right) \leftarrow\left(\mathbf{S}^{\dagger} \mathbf{V}\right)^{-1}$.

4. $\phi \leftarrow-\beta+\pi$.

$$
\begin{aligned}
& \rho_{0} \leftarrow \begin{cases}1, & \frac{r_{2}}{r_{0}} \leq 1 \\
\frac{r_{2}}{r_{0}}, & \frac{r_{2}}{r_{0}}>1 .\end{cases} \\
& \rho_{1} \leftarrow \begin{cases}1, & \frac{r_{2}}{r_{1}} \leq 1 \\
\frac{r_{2}}{r_{1}}, & \frac{r_{2}}{r_{1}}>1 .\end{cases}
\end{aligned}
$$$$
\text { 5. } \quad \mathbf{w}_{0} \leftarrow \mathbf{V R}\left(\begin{array}{c}
\rho_{0} \\
\rho_{1} e^{j \phi}
\end{array}\right) \text {. }
$$

The matrix inversion in Step 2 contains most of the complexity of the algorithm. Therefore, the algorithm has the same order of complexity as the MVDR beamformer. Since the constraint is loosened, the feasible set of the two-point quadratic constraint problem in (13) is a superset of the feasible set of the original problem in (11). The minimum found in this problem is a lowerbound of the minimum of the original problem. If the solution $\mathbf{w}_{0}$ in the two-point quadratic constraint problem in
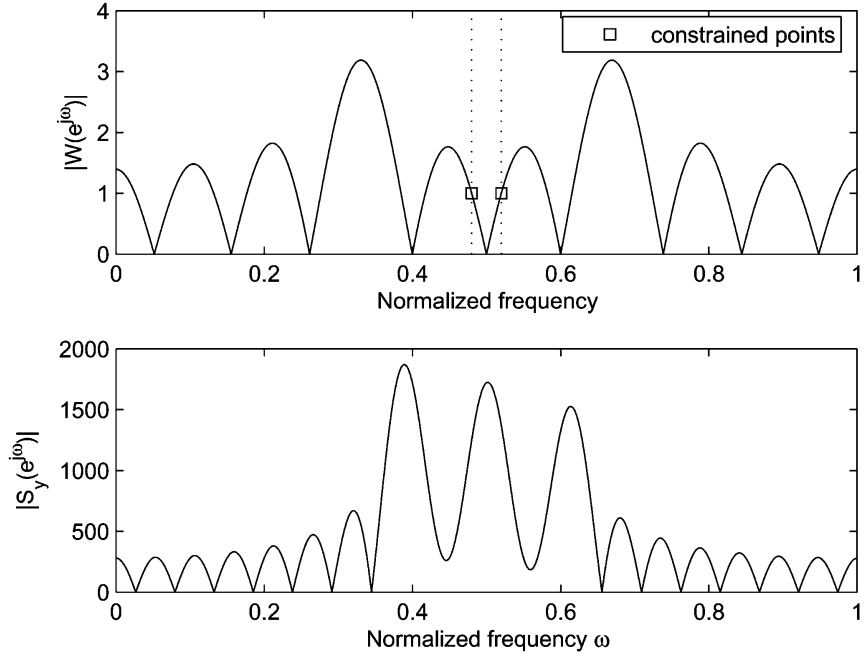

Fig. 2. Example of a solution of the two-point quadratic constraint problem that does not satisfy $\left|\mathbf{s}^{\dagger} \mathbf{w}\right| \geq 1$ for $\theta_{1} \leq \theta \leq \theta_{2}$.

(13) happens to satisfy the original constraint $\left|\mathbf{s}^{\dagger}(\theta) \mathbf{w}_{0}\right|^{2} \geq 1$ for $\theta_{1} \leq \theta \leq \theta_{2}$, then $\mathbf{w}_{0}$ is exactly the solution to the original problem in (11). The example provided in Fig. 1 is actually found by using the two-point quadratic constraint instead of the original constraint, but it also satisfies the original constraint. This makes it exactly the solution to the original problem in (11).

Unfortunately, in general, the original constraint $\left|\mathbf{s}^{\dagger}(\theta) \mathbf{w}\right| \geq$ 1 for $\theta_{1} \leq \theta \leq \theta_{2}$ is not guaranteed to be satisfied by the solution of the two-point quadratic constraint problem in (13). Fig. 2 shows an example where the original constraint is not satisfied. This example is obtained by increasing the power of the SOI in the example in Fig. 1. One can compare $\left|S_{y}\left(e^{j \omega}\right)\right|$ in Figs. 1 and 2 and find that the SOI power is much stronger in Fig. 2. In this case, the beamformer tends to put a zero between $\theta_{1}$ and $\theta_{2}$ to suppress the strong SOI. This makes $\left|W\left(e^{j \omega}\right)\right| \leq$ 1 for some $\omega$ between $\omega_{1}$ and $\omega_{2}$. The original constraint is thus not satisfied. This problem will be overcome by a method provided in Section V.

\section{Two-Point Quadratic Constraint With Diagonal Loading}

In Fig. 2, we observe that the energy of $\mathbf{w},\|\mathbf{w}\|^{2}=$ $\int_{0}^{2 \pi}\left|W\left(e^{j \omega}\right)\right|^{2} d \omega /(2 \pi)$ is quite large compared to that in Fig. 1. Fig. 3 shows the locations of the zeroes of the $z$-transform $W(z)$ of the beamformer in Fig. 2. One can observe that there is a zero between $\theta_{1}$ and $\theta_{2}$. This zero causes the signal cancellation in Fig. 2. It can be observed that the zero is very close to those two points which are constrained to have magnitudes greater than unity. When a zero is close to these quadratically constrained points, it attenuates the gain at these points. However, the magnitude responses at these points are constrained to exceed unity. To satisfy the constraints, the overall energy of $\mathbf{w}$ must be adjusted to a certain high level. Therefore, if a zero is between $\theta_{1}$ and $\theta_{2}$, which occurred in Fig. 3 , the norm of the weighting vector $\|\mathbf{w}\|$ will become very large. By using this fact, we can impose some penalty on $\|\mathrm{w}\|^{2}$ to force the zeroes between $\theta_{1}$ and $\theta_{2}$ to go away. This can be 


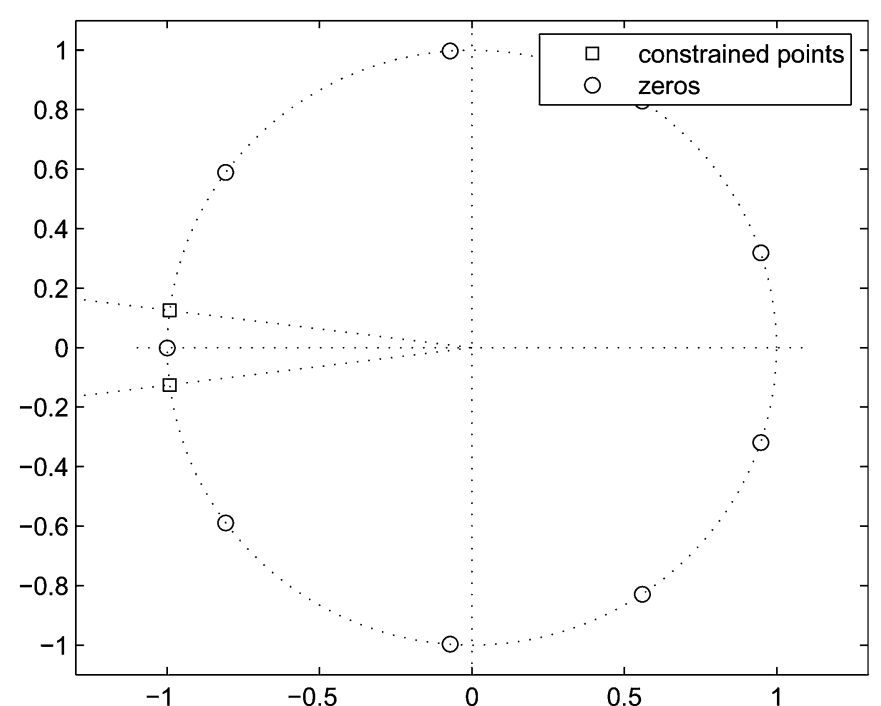

Fig. 3. Locations of zeroes of the beamformer in Fig. 2

accomplished by the diagonal loading approach mentioned in Section III-A. The corresponding optimization problem can be written as

$$
\begin{aligned}
\mathbf{w}_{\gamma}= & \arg \min _{\mathbf{w}} \mathbf{w}^{\dagger} \mathbf{R}_{\mathbf{y}} \mathbf{w}+\gamma\|\mathbf{w}\|^{2} \\
& \text { subject to }\left|\mathbf{s}^{\dagger}\left(\theta_{1}\right) \mathbf{w}\right| \geq 1 \text { and }\left|\mathbf{s}^{\dagger}\left(\theta_{2}\right) \mathbf{w}\right| \geq 1
\end{aligned}
$$

where $\gamma$ is the diagonal loading factor which represents the amount of the penalty put on $\|\mathbf{w}\|^{2}$. The solution $\mathbf{w}_{\gamma}$ can be found by performing the following modification on the output covariance matrix:

$$
\mathbf{R}_{\mathbf{y}} \leftarrow \mathbf{R}_{\mathbf{y}}+\gamma \mathbf{I}_{N}
$$

and then applying Algorithm 1. When $\gamma \rightarrow \infty$, the solution converges to

$$
\begin{aligned}
\mathbf{w}_{\infty}= & \arg \min _{\mathbf{w}}\|\mathbf{w}\|^{2} \\
& \text { subject to }\left|\mathbf{s}^{\dagger}\left(\theta_{1}\right) \mathbf{w}\right| \geq 1 \text { and }\left|\mathbf{s}^{\dagger}\left(\theta_{2}\right) \mathbf{w}\right| \geq 1 .
\end{aligned}
$$

The following lemma gives the condition for which $\mathbf{w}_{\infty}$ satisfies the constraint $\left|\mathbf{s}(\theta)^{\dagger} \mathbf{w}_{\infty}\right| \geq 1$ for all $\theta$ in $\theta_{1} \leq \theta \leq \theta_{2}$.

Lemma 1: $\left|\mathbf{s}^{\dagger}(\theta) \mathbf{w}_{\infty}\right| \geq 1$ for $\theta_{1} \leq \theta \leq \theta_{2}$ if and only if $\left|\sin \theta_{2}-\sin \theta_{1}\right| \leq \lambda /(d N)$.

Proof: According to (20), substituting $\mathbf{R}_{\mathbf{y}}=\mathbf{I}_{N}$ and applying Algorithm 1, one can obtain

$$
\begin{aligned}
\mathbf{w}_{\infty}= & \frac{1}{N+\left|\operatorname{sincd}\left(\frac{\omega_{2}-\omega_{1}}{2}\right)\right|} \\
& \times\left(\mathbf{s}\left(\theta_{1}\right)+\mathbf{s}\left(\theta_{2}\right) e^{j\left(\omega_{2}-\omega_{1}\right)(N-1) / 2}\right), \\
\omega_{1} \triangleq & \frac{2 \pi}{\lambda} d \sin \theta_{1}, \quad \omega_{2} \triangleq \frac{2 \pi}{\lambda} d \sin \theta_{2}, \\
\text { and } \operatorname{sincd}(\omega) \triangleq & \frac{\sin (\omega N)}{\sin \omega} .
\end{aligned}
$$

By direct substitution, one can obtain

$$
\left|\mathbf{s}^{\dagger}(\theta) \mathbf{w}_{\infty}\right|=\left|\frac{\operatorname{sincd}\left(\frac{\omega_{1}-\omega}{2}\right)+a \cdot \operatorname{sincd}\left(\frac{\omega_{2}-\omega}{2}\right)}{N+\left|\operatorname{sincd}\left(\frac{\omega_{2}-\omega_{1}}{2}\right)\right|}\right|
$$

where $\omega \triangleq 2 \pi / \lambda d \sin \theta$ and

$$
a= \begin{cases}1, & \text { if } \operatorname{sincd}\left(\frac{\omega_{2}-\omega_{1}}{2}\right)>0 \\ -1, & \text { otherwise. }\end{cases}
$$

By (21), it can be verified that

$$
\left|\mathbf{s}^{\dagger}(\theta) \mathbf{w}_{\infty}\right| \geq 1 \text { for } \omega_{1} \leq \omega \leq \omega_{2}
$$

if and only if

$$
\left|\omega_{2}-\omega_{1}\right| \leq \frac{2 \pi}{N}
$$

which can also be expressed as $\left|\sin \theta_{2}-\sin \theta_{1}\right| \leq \lambda /(d N)$.

If the condition $\left|\sin \theta_{1}-\sin \theta_{2}\right| \leq \lambda /(d N)$ is satisfied, $\gamma>0$ exists such that the condition $\left|\mathbf{s}^{\dagger}(\theta) \mathbf{w}_{\gamma}\right| \geq 1$ for $\theta_{1} \leq \theta \leq \theta_{2}$ is satisfied. For example, if $d=\lambda / 2, N=10, \theta_{1}=35^{\circ}$, and $\theta_{2}=55^{\circ}$, then we have

$$
\left|\sin \left(55^{\circ}\right)-\sin \left(35^{\circ}\right)\right| \approx 0.1824 \leq \frac{\lambda}{d N}=0.2 .
$$

In this case, $\gamma>0$ exists so that the robust condition $\left|\mathbf{s}^{\dagger}(\theta) \mathbf{w}_{\gamma}\right| \geq 1$ for $35^{\circ} \leq \theta \leq 55^{\circ}$ is satisfied. However, introducing the diagonal loading changes, the objective function $\mathbf{w}^{\dagger} \mathbf{R}_{\mathbf{y}} \mathbf{w}$ to $\mathbf{w}^{\dagger}\left(\mathbf{R}_{\mathbf{y}}+\gamma \mathbf{I}_{N}\right) \mathbf{w}$. The modification of the objective function affects the suppression of the interferences. To keep the objective function correct, $\gamma$ should be chosen as small as possible while the condition $\left|\mathbf{s}^{\dagger}(\theta) \mathbf{w}\right| \geq 1$ for $\theta_{1} \leq \theta \leq \theta_{2}$ is satisfied. For finding such a $\gamma$, we propose the following algorithm.

\section{Algorithm 2}

Given $\theta_{1}, \theta_{2}, \mathbf{R}_{\mathbf{y}}$, an initial value of $\gamma$, a search step size $\alpha>1$, and a set of angles $\zeta_{i}, i=1,2, \ldots, n$ which satisfies $\theta_{1}<\zeta_{i}<\theta_{2}$ for all $i, \mathbf{w}_{\gamma}$ can be computed by the following steps:

1. $\mathbf{R}_{\mathbf{y}} \leftarrow \mathbf{R}_{\mathbf{y}}+\gamma \mathbf{I}_{N}$.

2. Compute $\mathbf{w}_{\gamma}$ by Algorithm 1 .

3. If $\left|\mathbf{s}^{\dagger}\left(\zeta_{i}\right) \mathbf{w}_{\gamma}\right| \geq 1$ for all $i=1,2, \ldots, n$ then stop. else $\gamma \leftarrow \alpha \gamma$, and go to 1 .

Fig. 4 illustrates how Algorithm 2 works. In this figure, the set $\mathcal{A}=\left\{\mathbf{w}|| \mathbf{s}^{\dagger}(\theta) \mathbf{w} \mid \geq 1, \theta=\theta_{1}, \theta_{2}\right\}$ is the feasible set of the two-point quadratic constraint problem in (13). The set $\mathcal{B}=\left\{\mathbf{w}|| \mathbf{s}^{\dagger}(\theta) \mathbf{w} \mid \geq 1, \theta_{1} \leq \theta \leq \theta_{2}\right\}$ is the feasible set of the mismatched steering vector problem in (11). If the condition $\left|\sin \theta_{1}-\sin \theta_{2}\right| \leq \lambda /(d N)$ is satisfied, Lemma 1 shows that $\mathbf{w}_{\infty} \in \mathcal{B}$. In this case, $\gamma>0$ exists so that $\mathbf{w}_{\gamma} \in \mathcal{B}$. Algorithm 2 keeps increasing $\gamma$ by multiplying $\alpha$ until $\left|\mathbf{s}^{\dagger}\left(\zeta_{i}\right) \mathbf{w}_{\gamma}\right| \geq 1$ 
for all $i=1,2, \ldots, n$ is satisfied. This is an approximation for $\mathbf{w}_{\gamma} \in \mathcal{B}$. The number $n$ can be very small. In Section $\mathrm{V}, n=3$ works well for all of the cases. Also, the SINR is not sensitive to the choice of $\alpha$, as we will see later.

\section{NUMERICAL EXAMPLES}

For the purpose of design examples, the same parameters used in [8] are used in this section. A uniform linear array (ULA) of $N=10$ omnidirectional sensors spaced a half-wavelength apart (i.e., $d=\lambda / 2$ ) is considered. There are three signals impinging upon this array, as follows:

1) the SOI $x(t)$ with an angle of arrival $\theta$;

2) an interference signal $x_{\text {int } 1}(t)$ with an angle of arrival $\theta_{\text {int } 1}=30^{\circ}$

3 ) another interference signal $x_{\mathrm{int} 2}(t)$ with an angle of arrival $\theta_{\text {int2 }}=75^{\circ}$.

The received narrowband array output can be modeled as

$$
\mathbf{y}(t)=x(t) \mathbf{s}(\theta)+x_{\text {int } 1}(t) \mathbf{s}\left(\theta_{\text {int } 1}\right)+x_{\text {int2 }}(t) \mathbf{s}\left(\theta_{\text {int } 2}\right)+\mathbf{n}(t)
$$

where $\mathbf{s}(\theta)$ is the steering vector defined in (1), and $\mathbf{n}(t)$ is the noise. We assume $x(t), x_{\mathrm{int} 1}(t), x_{\mathrm{int} 2}(t)$, and $\mathbf{n}(t)$ are the zeromean wide-sense stationary random process satisfying

$$
\begin{aligned}
E\left[\mathbf{n}(t) \mathbf{n}^{\dagger}(t)\right] & =\mathbf{I}_{N} \\
E\left[|x(t)|^{2}\right] & =\sigma_{x}^{2}=\mathrm{SNR} \cdot 1 \\
E\left[\left|x_{\text {int1 }}(t)\right|^{2}\right] & =\sigma_{\text {int1 }}^{2}=10^{4}(40 \mathrm{~dB} \text { above noise }) \\
E\left[\left|x_{\text {int } 2}(t)\right|^{2}\right] & =\sigma_{\text {int2 }}^{2}=10^{2}(20 \mathrm{~dB} \text { above noise }) .
\end{aligned}
$$

Thus, the covariance matrix of the narrowband array output $\mathbf{y}(t)$ can be expressed as

$$
\begin{aligned}
\mathbf{R}_{\mathbf{y}} & \triangleq E\left[\mathbf{y}(t) \mathbf{y}^{\dagger}(t)\right] \\
& =\sigma_{x}^{2} \mathbf{s}(\theta) \mathbf{s}^{\dagger}(\theta)+\sum_{i=1}^{2} \sigma_{\text {int }, \mathrm{i}}^{2} \mathbf{s}\left(\theta_{\text {int }, \mathrm{i}}\right) \mathbf{s}^{\dagger}\left(\theta_{\text {int }, \mathrm{i}}\right)+\mathbf{I}_{N} .
\end{aligned}
$$

1) Example 1: SINR versus diagonal loading factor $\gamma$.

In this example, the actual arrival angle $\theta$ is $43^{\circ}$, but the assumed arrival angle $\theta_{m}$ is $45^{\circ}$. The SINR defined in (2) is compared for a different diagonal loading factor $\gamma$. The following five methods involving diagonal loading are considered:

1) Algorithm 1 in Section IV with the new method with $\theta_{1}=$ $42^{\circ}$ and $\theta_{2}=48^{\circ}$;

2) general-rank method [9] in (10) with the parameter

$$
\epsilon=\max _{48^{\circ} \geq \theta \geq 42^{\circ}}\left\|\mathbf{s}(\theta) \mathbf{s}^{\dagger}(\theta)-\mathbf{s}\left(45^{\circ}\right) \mathbf{s}^{\dagger}\left(45^{\circ}\right)\right\|_{F} \approx 4.73 ;
$$

3) diagonal loading method [11], [12] in Section III-A;

4) directional LCMV [15], [16] with two linear constraints which forces the responses of the signals from $42^{\circ}$ and $48^{\circ}$ to be unity;

5) derivative LCMV [17]-[19] with two linear constraints which forces the responses of the signals from $45^{\circ}$ to be unity and the derivative of the beampattern on $45^{\circ}$ to be zero.

The SINR of the MVDR beamformer without mismatch is also plotted. This is an upperbound on the SINR. Fig. 5 shows the result for SNR $=10 \mathrm{~dB}$. One can observe that there is a huge jump in the SINR of Algorithm 1 around $\gamma=3$. When this occurs,

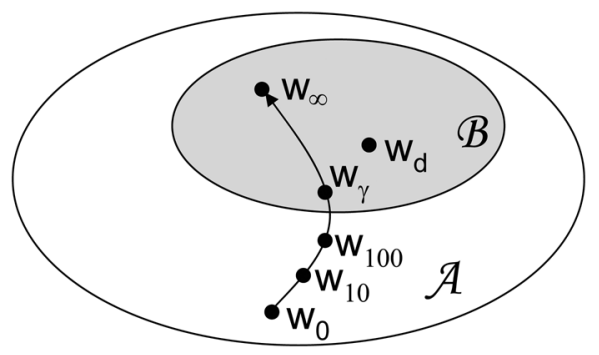

Fig. 4. Illustration of Algorithm 2, where $\mathcal{A}=\left\{\mathbf{w}|| \mathbf{s}^{\dagger}(\theta) \mathbf{w} \mid \geq 1, \theta=\right.$ $\left.\theta_{1}, \theta_{2}\right\}$ and $\mathcal{B}=\left\{\mathbf{w}|| \mathbf{s}^{\dagger}(\theta) \mathbf{w} \mid \geq 1, \theta_{1} \leq \theta \leq \theta_{2}\right\}$.

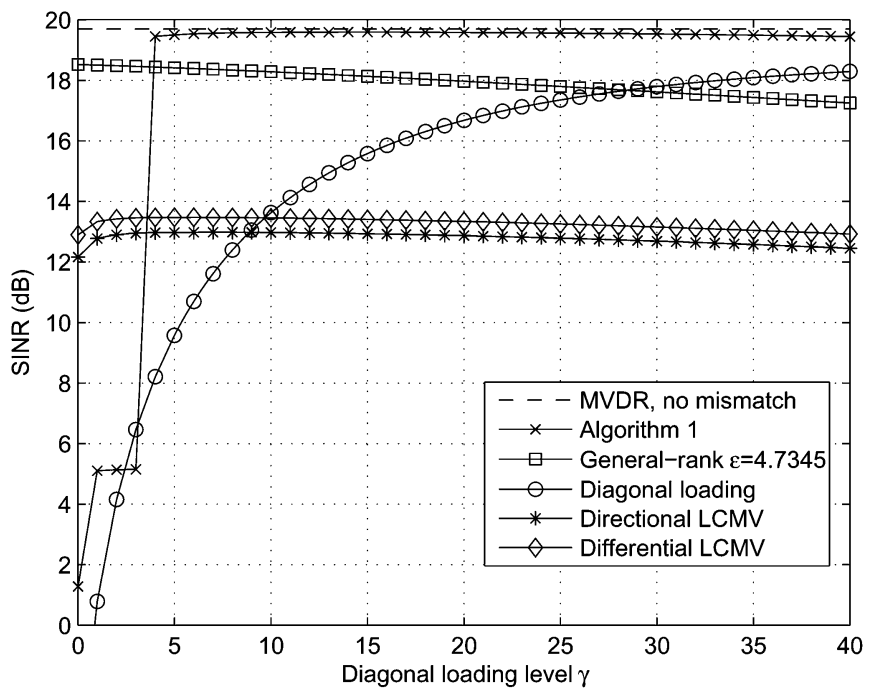

Fig. 5. Example 1: SINR versus $\gamma$ for $\mathrm{SNR}=10 \mathrm{~dB}$.

the SINR of Algorithm 1 increases significantly and becomes very close to the upperbound provided by the MVDR beamformer without mismatch. This jump occurs when the beampattern changes from Fig. 2 to Fig. 1. Once the beamformer enters the set $\mathcal{B}$ as illustrated in Fig. 4, the SINR increases dramatically. After that, the SINR decays slowly as $\gamma$ increases because of the oversuppression of white noise. Fig. 6 shows the case of $\mathrm{SNR}=20 \mathrm{~dB}$. For large SNR, larger $\gamma$ is needed for the beamformer to be in set $\mathcal{B}$. Observing Figs. 5 and 6, we can see why Algorithm 2 works so well. Algorithm 2 increases $\gamma$ by repeatedly multiplying $\alpha$ until $\mathbf{w}_{\gamma}$ satisfies $\left|\mathbf{w}_{\gamma}^{\dagger} \mathbf{s}\left(\zeta_{i}\right)\right| \geq 1$ for $i=$ $1,2, \ldots, n$. This occurs as $\gamma$ crosses the jump in SINR. Also, the SINR is not sensitive to the choice of $\alpha$ because the SINR decays very slowly after the jump. By Algorithm 2, we can find a suitable $\gamma$ with only a few iterations. For other approaches involving diagonal loading, it is not clear how to find a good diagonal loading factor $\gamma$. One can observe that Algorithm 1 has a very different SINR performance than the two-point directional LCMV with diagonal loading. This shows that further optimization of the parameters $\phi, \rho_{0}$, and $\rho_{1}$ in Section IV-B is very crucial.

2) Example 2: SINR versus SNR.

In this example, the actual arrival angle $\theta$ is $43^{\circ}$, but the assumed arrival angle $\theta_{m}$ is $45^{\circ}$. The SINRs in (2) are compared for different SNRs ranging from -20 to $30 \mathrm{~dB}$. The following methods are considered. 


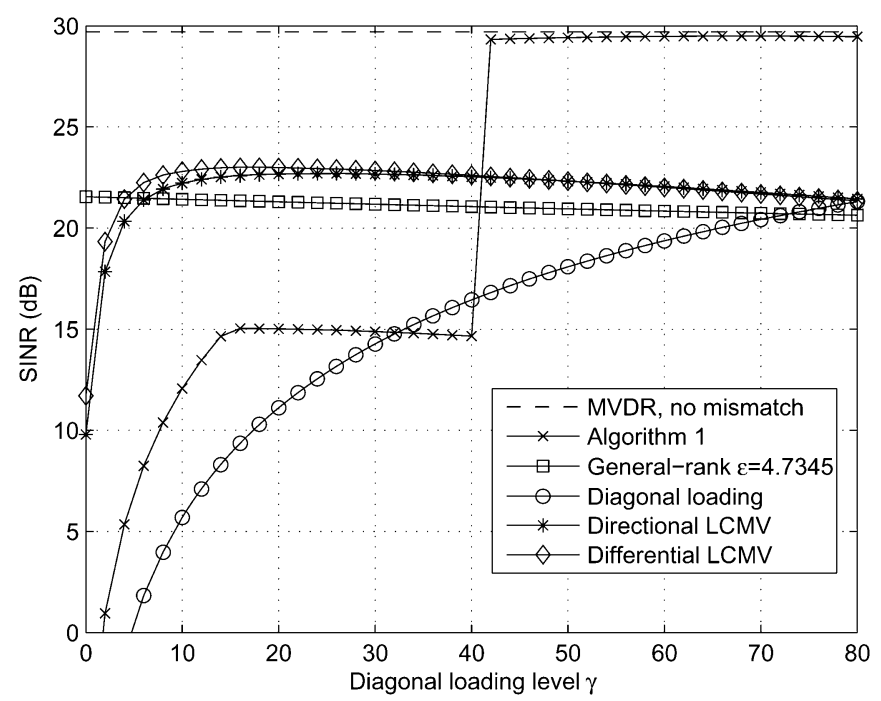

Fig. 6. Example 1 continued: SINR versus $\gamma$ for $\mathrm{SNR}=20 \mathrm{~dB}$.

1) Algorithm 2 with $\theta_{1}=42^{\circ}, \theta_{2}=48^{\circ}, \zeta_{1}=43.5^{\circ}$, $\zeta_{2}=45^{\circ}, \zeta_{3}=46.5^{\circ}$, initial $\gamma=1$, and step size $\alpha=2$;

2) general-rank method-same as in Example 1;

3) extended diagonal loading method [6]-[8] in (8) with the parameter

$$
\epsilon=\max _{48^{\circ} \geq \theta \geq 42^{\circ}}\left\|\mathbf{s}(\theta)-\mathbf{s}\left(45^{\circ}\right)\right\| \approx 1.95 ;
$$

the algorithm in [7] is used to compute the diagonal loading level;

4) directional LCMV [15], [16] with two linear constraints which forces the responses of the signals from $42^{\circ}$ and $48^{\circ}$ to be unity;

5) directional LCMV with three linear constraints at the angles $42^{\circ}, 45^{\circ}$, and $48^{\circ}$;

6) derivative LCMV with two linear constraints which force the responses of the signals from $45^{\circ}$ to be unity and the derivative of the beampattern on $45^{\circ}$ to be zero;

7) derivative LCMV with three linear constraints which force the responses of the signals from $45^{\circ}$ to be unity and both the first and second derivatives of the beampattern on $45^{\circ}$ to be zero;

8) the standard MVDR beamformer in (5).

Due to the fact that no finite-sample effect is considered, except in Algorithm 2 and the extended diagonal loading method, no diagonal loading has been used in these methods. Again, the SINR of the MVDR beamformer without mismatch is also plotted as a benchmark. The results are shown in Fig. 7. The SINR of the standard MVDR beamformer is seriously degraded with only $2^{\circ}$ of mismatch. When the SNR increases, the MVDR beamformer tends to suppress the strong SOI to minimize the total output variance. Therefore, in the high SNR region, the SINR decreases when SNR increases. The LCMV beamformers have good performances in the high SNR region. However, the performance in the low SNR region is much worse compared to other methods. This is because the linear equality constraints are too strong compared to the quadratic inequality constraints. One can observe that for both directional and derivative LCMV

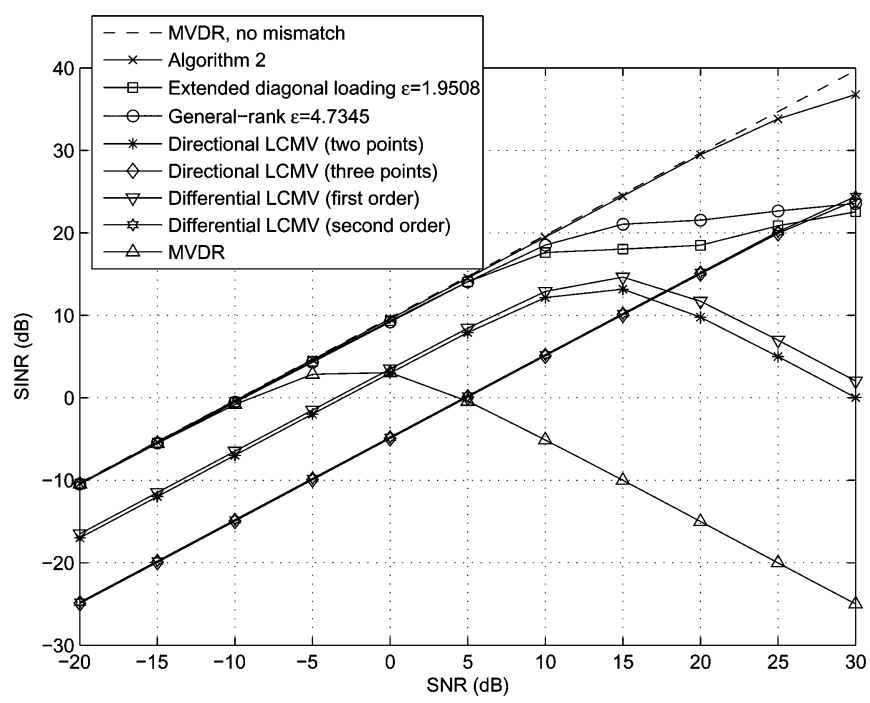

Fig. 7. Example 2: SINR versus SNR.

methods, each extra linear constraint decreases the SINR by about the same amount in the low SNR region. In this example, Algorithm 2 has the best SINR performance. It is very close to the upperbound provided by the MVDR beamformer without mismatch. Algorithm 2 has better SINR performance than the general rank method [9] and the extended diagonal loading method [6]-[8] because the uncertainty set has been simplified to be robust only against DOA mismatch. Note that even though these methods have worse performances than Algorithm 2 with regard to DOA error, they have the advantages of robustness against more general types of steering vector mismatches. The number of iterations in Algorithm 2 depends on the SNR and the choice of $\alpha$. For instance, it converges with two steps when SNR $=10 \mathrm{~dB}$ and six steps when SNR $=20 \mathrm{~dB}$ in this example.

3) Example 3: SINR versus mismatch angle.

In this example, the assumed signal arrival angle $\theta_{m}$ is $45^{\circ}$, and the actual arrival angle ranges from $\theta=41^{\circ}$ to $\theta=49^{\circ}$. The SINR in (2) is compared for different mismatched angles $\left(\theta-\theta_{m}\right)$. The following methods are considered:

1) Algorithm 2 with $\theta_{1}=41^{\circ}, \theta_{2}=49^{\circ}, \zeta_{1}=43^{\circ}, \zeta_{2}=$ $45^{\circ}, \zeta_{3}=47^{\circ}$, initial $\gamma=1$, and step size $\alpha=2$;

2) general-rank method [9] in (10) with the parameter

$$
\epsilon=\max _{49^{\circ} \geq \theta \geq 41^{\circ}}\left\|\mathbf{s}(\theta) \mathbf{s}^{\dagger}(\theta)-\mathbf{s}\left(45^{\circ}\right) \mathbf{s}^{\dagger}\left(45^{\circ}\right)\right\|_{F} \approx 6.25 ;
$$

3) extended diagonal loading method [6]-[8] in (8) with the parameter

$$
\epsilon=\max _{49^{\circ} \geq \theta \geq 41^{\circ}}\left\|\mathbf{s}(\theta)-\mathbf{s}\left(45^{\circ}\right)\right\| \approx 2.56 ;
$$

4) directional LCMV [15], [16] with three linear constraints which forces the responses of the signal from $41^{\circ}, 45^{\circ}$, and $49^{\circ}$ to be unity;

5) first-order derivative LCMV—-same as in Example 1;

6) the standard MVDR beamformer in (5).

The SINR of the MVDR beamformer without mismatch is also displayed in the following figures. The results for SNR = $0 \mathrm{~dB}$ are shown in Fig. 8, and the results for SNR $=10 \mathrm{~dB}$ 


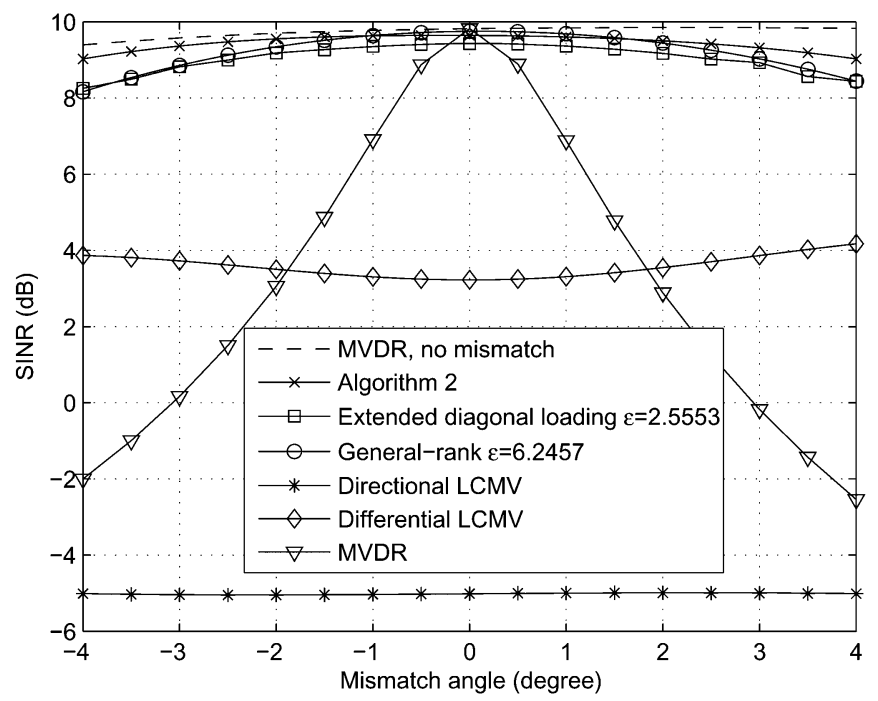

Fig. 8. Example 3: SINR versus mismatch angle for $\mathrm{SNR}=0 \mathrm{~dB}$.

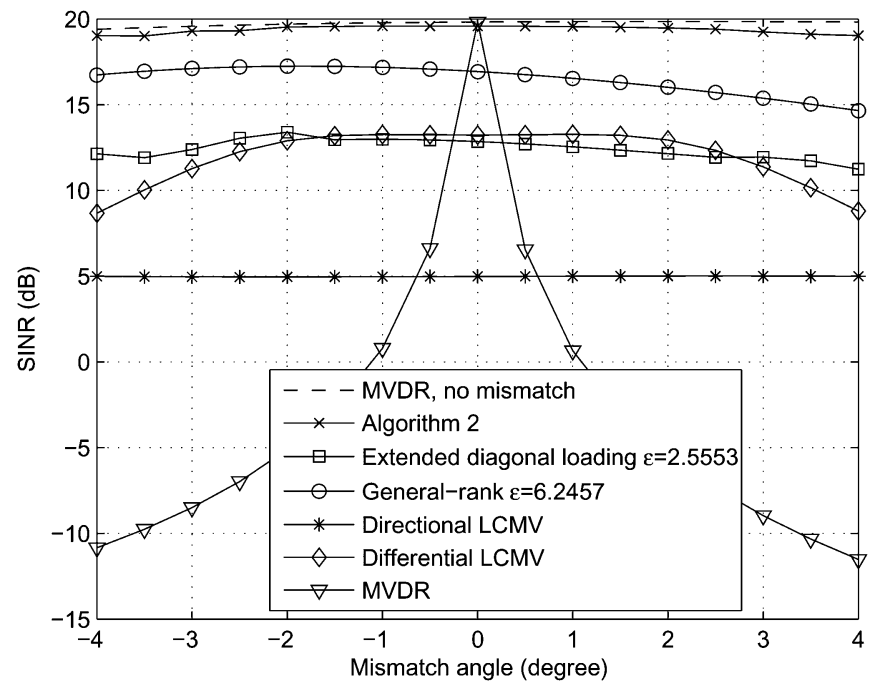

Fig. 9. Example 3 continued: SINR versus mismatch angle for $\mathrm{SNR}=10 \mathrm{~dB}$.

are shown in Fig. 9. One can observe that the standard MVDR beamformer is very sensitive to the arrival angle mismatch. It is more sensitive when the SNR is larger. Except for the standard MVDR, these methods maintain steady SINRs with the mismatched angle $\theta-\theta_{m}$ varying. In this example, Algorithm 2 has the best SINR performance among these methods. Moreover, when there is no mismatch, the SINR of Algorithm 2 decreases slightly compared to the standard MVDR beamformer.

4) Example 4: SINR versus $N$.

In this example, the SINR is being compared for various numbers of antennas $N$. The actual angle of arrival $\theta$ is $43^{\circ}$, but the assumed angle of arrival $\theta_{m}$ is $45^{\circ}$. The following methods are considered:

1) Algorithm 2-the same as in Example 2;

2) general-rank method-same as in Example 2 except $\epsilon$ is now a function of $N$, and it can be expressed as

$$
\epsilon(N)=\max _{48^{\circ} \geq \theta \geq 42^{\circ}}\left\|\mathbf{s}(\theta) \mathbf{s}^{\dagger}(\theta)-\mathbf{s}\left(45^{\circ}\right) \mathbf{s}^{\dagger}\left(45^{\circ}\right)\right\|_{F} ;
$$

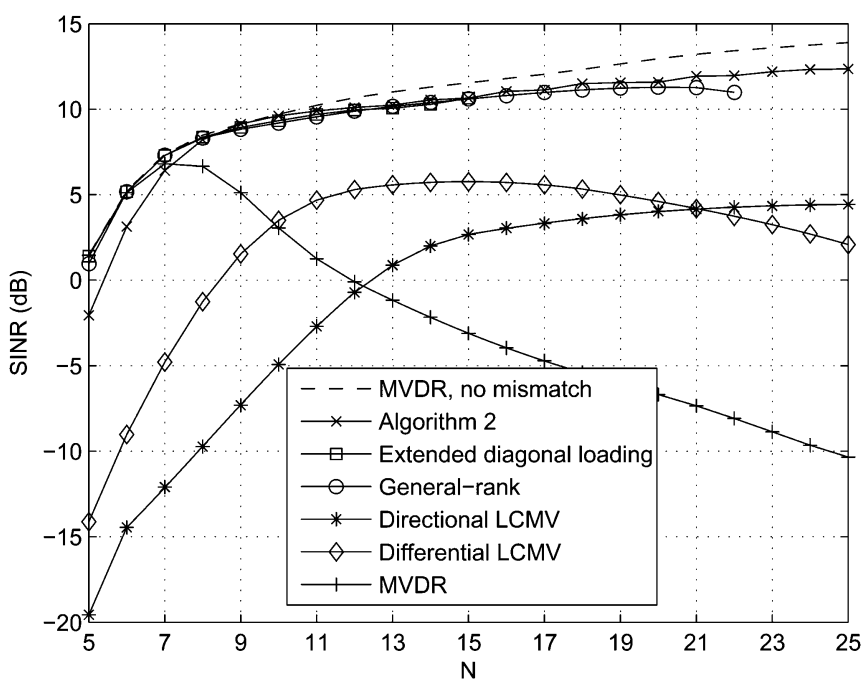

Fig. 10. Example 4: SINR versus a number of antennas for $\mathrm{SNR}=0 \mathrm{~dB}$.

3) extended diagonal loading method-same as in Example 2 except $\epsilon$ is now a function of $N$, and it can be expressed as

$$
\epsilon(N)=\max _{48^{\circ} \geq \theta \geq 42^{\circ}}\left\|\mathbf{s}(\theta)-\mathbf{s}\left(45^{\circ}\right)\right\|
$$

4) three-point directional LCMV method-same as in Example 2;

5) first-order derivative LCMV - same as in Example 2;

6) the standard MVDR beamformer in (5).

The results for the case of SNR $=0 \mathrm{~dB}$ and $\mathrm{SNR}=10 \mathrm{~dB}$ are shown in Figs. 10 and 11, respectively. One can observe that when there is no mismatch, the SINR performance of the MVDR beamformer is an increasing function of the number of the antennas $N$, since the beamformer has a better ability to suppress the interferences and noise when $N$ increases. However, for the MVDR beamformer with mismatch, the beamformer has a better ability to suppress the SOI as well as interferences when $N$ increases. Therefore, the SINR of the MVDR beamformer increases at the beginning and then decays rapidly when $N$ increases. For the general rank method, the SINRs when $N$ is larger than 22 are discarded because the corresponding $\epsilon$ are greater than $\left\|\mathbf{s}(\theta) \mathbf{s}^{\dagger}(\theta)\right\|_{F}$. For the same reason, the SINRs when $N$ is larger than 15 are discarded in the extended diagonal loading method. Again, in this example, Algorithm 2 has very good performance. Among all of the robust beamformers, only Algorithm 2 has nondecreasing SINR with respect to $N$. However, this does not mean there is no limitation on $N$ for Algorithm 2. According to Lemma 1, the condition which guarantees the convergence of Algorithm 2 can be expressed as

$$
\left|\sin \left(48^{\circ}\right)-\sin \left(42^{\circ}\right)\right| \approx .074<\frac{\lambda}{N d}=\frac{2}{N} \Rightarrow N \leq 27 .
$$

This means that if the number of antennas $N$ is larger than 27, Algorithm 2 is not guaranteed to converge. In this example, Algorithm 2 fails to converge when $N=28$.

5) Example 5: SINR versus number of snapshots. 


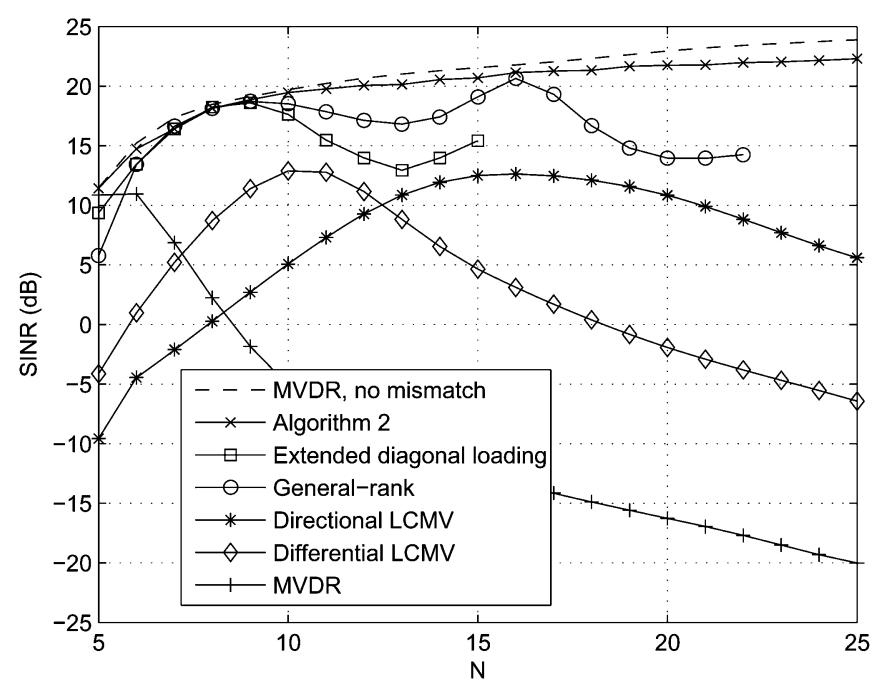

Fig. 11. Example 4 continued: SINR versus number of antennas for SNR $=10 \mathrm{~dB}$.

The covariance matrices $\mathbf{R}_{\mathbf{y}}$ used in the previous examples are assumed to be perfect. In practice, the covariance matrix can only be estimated. For example, we can use

$$
\hat{\mathbf{R}}_{\mathbf{y}}(K)=\frac{1}{K} \sum_{k=1}^{K} \mathbf{y}(k T) \mathbf{y}^{\dagger}(k T)
$$

where $T$ is the sampling rate of the array, and $K$ is the number of snapshots. The accuracy of the estimated covariance matrix $\hat{\mathbf{R}}_{\mathbf{y}}$ affects the SINR of the beamformer. In this example, the actual arrival angle is $43^{\circ}$, but the assumed arrival angle is $45^{\circ}$. The SINRs are compared for a different number of snapshots $K$. The following methods are considered:

1) Algorithm 2 with $\theta_{1}=42^{\circ}, \theta_{2}=48^{\circ}, \zeta_{1}=43.5^{\circ}$, $\zeta_{2}=45^{\circ}, \zeta_{3}=46.5^{\circ}$, initial $\gamma=10$, and step size $\alpha=2$;

2) general-rank method [9] with $\epsilon \approx 4.73$ and $\gamma=10$;

3 ) extended diagonal loading method [6]-[8] with the parameter $\epsilon \approx 1.95$ before using the algorithm in [7] to compute the diagonal loading level, the estimated covariance matrix is first modified by $\hat{\mathbf{R}}_{\mathbf{y}} \leftarrow \hat{\mathbf{R}}_{\mathbf{y}}+10 \mathbf{I}_{\mathbf{N}}$; in other words, an initial diagonal loading level $\gamma=10$ is used;

4) three-point directional LCMV-same as in Example 2 except a diagonal loading level $\gamma=10$ is used;

5) first-order derivative LCMV—-same as in Example 2 except a diagonal loading level $\gamma=10$ is used;

6) fixed diagonal loading [11], [12] with $\gamma=10$;

7) the standard MVDR beamformer in (5) with correct steering vector $\mathbf{s}(\theta)$.

All of these methods use the estimated covariance matrix $\hat{\mathbf{R}}_{\mathbf{y}}(K)$. Due to the fact that the finite-sample effect is considered, each method uses an appropriate diagonal loading level. The SINR of the MVDR beamformer, which uses the correct steering vector $\mathbf{s}(\theta)$ and the perfect covariance matrix $\mathbf{R}_{\mathbf{y}}$, is used as an upperbound. In this example, noise $\mathbf{n}(k T)$ is generated according to the Gaussian distribution. The SINR is computed by using the averaged signal power and interference-plus-noise power over 1000 samples. The results are shown in Fig. 12 for SNR $=10 \mathrm{~dB}$. The MVDR beamformer

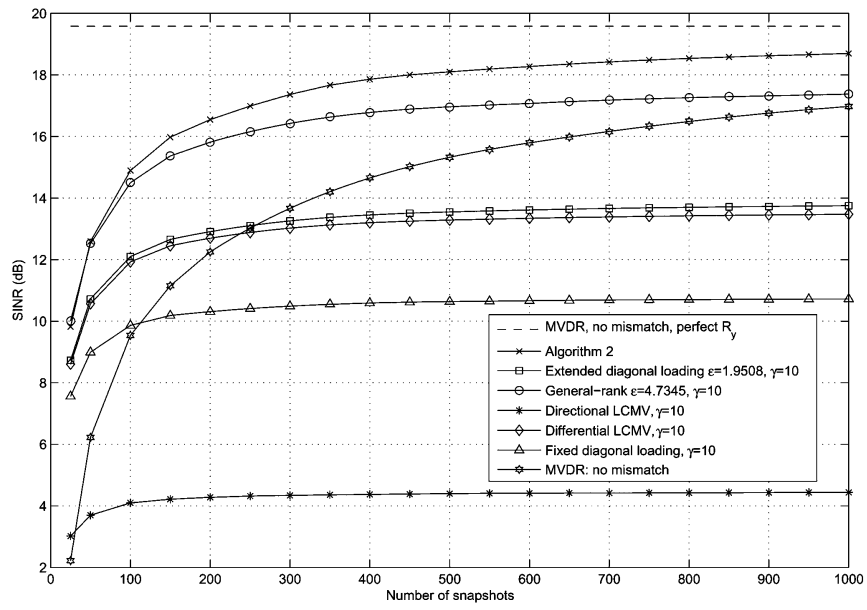

Fig. 12. Example 5: SINR versus number of snapshots for $\mathrm{SNR}=10 \mathrm{~dB}$.

without mismatch suffers from the finite-sample effect. Therefore, the SINR is low when the number of snapshots is small. For the fixed diagonal loading method, the SINR is relatively high when the number of snapshots is small. This shows that the diagonal loading method is effective against the finite-sample effect. However, SINR stops increasing after some number of snapshots because of the SOI steering vector mismatch. Again, Algorithm 2 has the best SINR performance for most situations. This shows that it is robust against both the finite-sample effect and the DOA mismatch.

The famous rapid convergence theorem proposed by Reed $e t$ al. in [27] states that an SINR loss of $3 \mathrm{~dB}$ can be obtained by using the number of snapshots $K$ equal to twice the number of antennas $N$. In this example, twice the number of antennas $N$ is only 20 . However, this result is applicable only to the case where the samples are not contaminated by the target signal. Therefore, it cannot be applied to this example. One can see that in Fig. 12, the SINR requires more samples to converge because the sampled covariance matrices contain the target signal of $10 \mathrm{~dB}$. In [24], the authors have pointed out that the sample covariance matrix error is equivalent to the DOA error. Since our method is designed for robustness against DOA mismatch, it is also robust against the finite-sample effect. However, it is not clear how to specify an appropriate uncertainty set to obtain the robustness against the finite-sample effect. This problem will be explored in future work.

The SOI power can be estimated by the total output variance $\mathbf{w}^{\dagger} \hat{\mathbf{R}}_{\mathbf{y}} \mathbf{w}$. Fig. 13 shows the corresponding estimated SOI power. One can see that the estimated SOI power converges much faster than the SINR. The estimated SOI power represents the sum of signal and "interference + noise" power but the SINR represents the ratio of them. The reduction of the interference plus noise is subtle in the estimated SOI power because it only changes a small portion of the total variance. However, the reduction of the interference plus noise can cause a significant change in SINR. A change in interference plus noise does not affect the SOI as much as it affects the SINR. Therefore, the estimated SOI power converges faster than the SINR.

6) Example 6: SINR versus SNR for general type mismatch. 


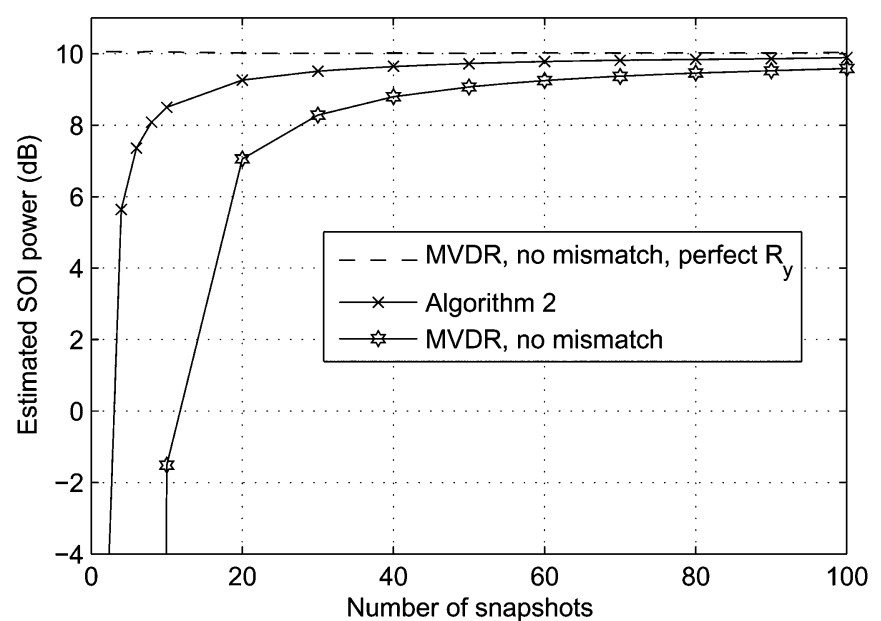

Fig. 13. Estimated SOI power versus the number of snapshots for SNR $=$ $10 \mathrm{~dB}$.

In the previous examples, we consider only the DOA mismatch. Although the proposed method is designed for solving only the DOA mismatch problem, in this example, we consider a more general type of mismatch. In this example, the mismatched steering vector is modeled as

$$
\mathbf{s}_{m}=\mathbf{s}(\theta)+\mathbf{e}
$$

where $\mathbf{e}$ is a random vector with i.i.d. components $e_{i} \sim$ $\mathcal{C N}\left(0, \sigma_{e}^{2}\right)$ for all $i$. In this example, $\sigma_{e}^{2}$ is chosen to be 0.01 . The SINRs in (2) are compared for different SNRs ranging from -20 to $30 \mathrm{~dB}$. The SINR are calculated by the averaged energy of more than 1000 samples. All parameters are as in Example 2 except the steering vector mismatch. The following methods are considered:

1) Algorithm 2 with $\theta_{1}=41^{\circ}, \theta_{2}=49^{\circ}, \zeta_{1}=43^{\circ}, \zeta_{2}=$ $45^{\circ}, \zeta_{3}=47^{\circ}$, initial $\gamma=1$, and step size $\alpha=2$;

2) general-rank method-same as in Example 2 except $\epsilon$ is chosen to be $4 N \sigma_{e}=4$ to cover most of the steering vector error;

3) extended diagonal loading method-same as in Example 2 except $\epsilon$ is chosen to be $2 N \sigma_{e}=2$ to cover most of the steering vector error;

4) two-point directional LCMV-same as in Example 2;

5) three-point directional LCMV_-same as in Example 2;

6) first-order derivative LCMV - same as in Example 2;

7) second-order derivative LCMV - same as in Example 2;

8) the standard MVDR beamformer in (5).

Due to the fact that no finite-sample effect is considered, except in Algorithm 2, and the extended diagonal loading method, no diagonal loading has been used in these methods. Again, the SINR of the MVDR beamformer without mismatch is also plotted as a benchmark. The results are shown in Fig. 14. The SINRs of the standard MVDR beamformer and all of the LCMV methods are seriously degraded by this general type mismatch in the high SNR region. However, the proposed algorithm still has good performance. As expected, the proposed algorithm has worse performance than the extended diagonal loading method when the SNR is equal to 0,10 , and $15 \mathrm{~dB}$ because it is designed for robustness against DOA mismatch. The differences are about $1.5 \mathrm{~dB}$. Surprisingly, however, it has a better SINR

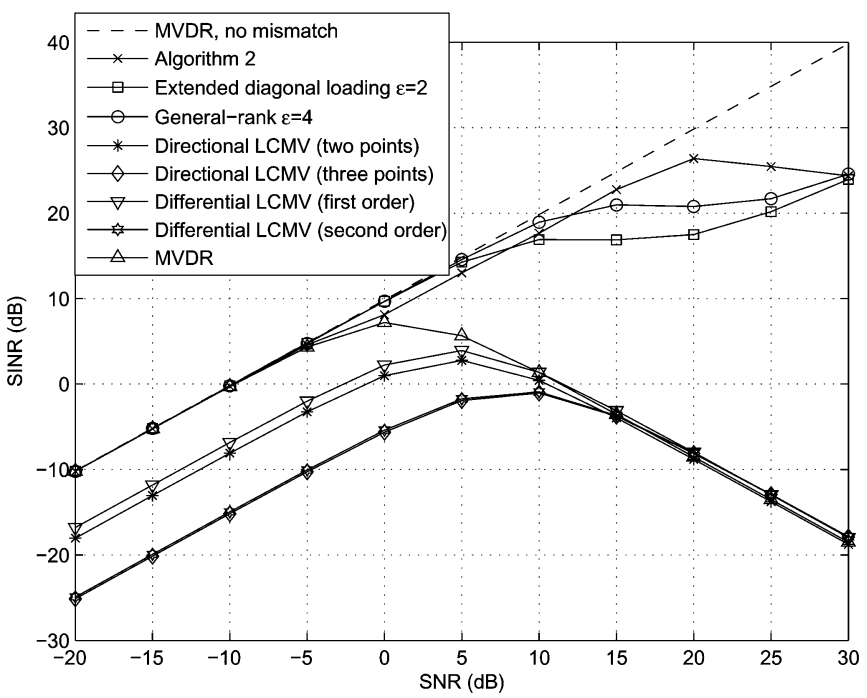

Fig. 14. Example 6: SINR versus SNR for general type mismatch.

performance in the high SNR region compared to other uncertainty-based methods. The authors' conjecture is that these uncertainty-based methods are based on worst case; however, the SINR is obtained by averaging the energy. The worst-case design guarantees that every time the SOI is protected; however, it does not guarantee that, in average, the SINR performance is good. In the worst-case sense, the extended diagonal loading method [6]-[8] should be the best choice. Nevertheless, this example shows that the proposed method has unexpected good performance compared to the LCMV methods when a general type of steering vector mismatches occurs. We believe that the proposed algorithm is a good candidate for robust beamforming when DOA mismatch is dominant.

\section{CONCLUSION}

In this paper, a new beamformer, which is robust against DOA mismatch, is introduced. This approach quadratically constrains the magnitude responses of two steering vectors and then uses a diagonal loading method to force the magnitude response at a range of arrival angles to exceed unity. Therefore, this method can always force the gains at a desired range of angles to exceed a constant level while suppressing the interference and noise. The analytic solution to the nonconvex quadratically constrained minimization problem has been derived, and the diagonal loading factor $\gamma$ can be determined by a simple iteration method proposed in Algorithm 2. This method is applicable to the point-source model where $\mathbf{s}(\theta)$ is known whenever $\theta$ is known. The complexity required in Algorithm 1 is approximately the same as in the MVDR beamformer. The overall complexity depends on the number of iterations in Algorithm 2 which depends on the SNR. In our numerical examples, when SNR $<10 \mathrm{~dB}$, the number of iterations is less than three. The numerical examples demonstrate that our approach has excellent SINR performance under a wide range of conditions.

\section{ACKNOWLEDGMENT}

The authors would like to express their deep appreciation to the reviewers who provided very useful criticism and many insightful remarks. 


\section{REFERENCES}

[1] J. Capon, "High-resolution frequency-wavenumber spectrum analysis," Proc. IEEE, vol. 57, no. 8, pp. 1408-1418, Aug. 1969.

[2] H. Cox, "Resolving power and sensitivity to mismatch of optimum array processors," J. Acoust. Soc. Amer., vol. 54, pp. 771-758, 1973.

[3] J. Li and P. Stoica, Eds., Robust Adaptive Beamforming. New York: Wiley, 2006.

[4] J. R. Guerci, Space-Time Adaptive Processing. Norwood, MA: Artech House, 2003.

[5] S. Q. Wu and J. Y. Zhang, "A new robust beamforming method with antennae calibration erros," in Proc. IEEE Wireless Commun. Networking Conf., New Orleans, LA, Sep. 1999, vol. 2, pp. 869-872.

[6] S. Vorobyov, A. B. Gershman, and Z.-Q. Luo, "Robust adaptive beamforming using worst-case performance optimization: A solution to the signal mismatch problem," IEEE Trans. Signal Process., vol. 51, no. 2, pp. 313-324, Feb. 2003.

[7] J. Li, P. Stoica, and Z. Wang, "On robust capon beamforming and diagonal loading," IEEE Trans. Signal Process., vol. 51, no. 7, pp. 1702-1714, Jul. 2003.

[8] R. G. Lorenz and S. P. Boyd, "Robust minimum variance beamforming," IEEE Trans. Signal Process., vol. 53, no. 5, pp. 1684-1696, May 2005.

[9] S. Shahbazpanahi, A. B. Gershman, Z.-Q. Luo, and K. M. Wong, "Robust adaptive beamforming for general-rank signal models," IEEE Trans. Signal Process., vol. 51, no. 9, pp. 2257-2269, Sep. 2003.

[10] J. L. Krolik, "The performance of matched-field beamformers with mediterranean vertical array data," IEEE Trans. Signal Process., vol. 44, no. 10, pp. 2605-2611, Oct. 1996.

[11] Y. I. Abramovich, "Controlled method for adaptive optimization of filters usisng the criteriion of maximum SNR," Radio Eng. Electron. Phys., vol. 26, pp. 87-95, Mar. 1981.

[12] B. D. Carlson, "Covariance matrix estimation errors and diagonal loading in adaptive arrays," IEEE Trans. Aerosp. Electron. Syst., vol. 24, no. 4, pp. 397-401, Jul. 1988.

[13] A. H. Booker, C. Y. Ong, J. P. Burg, and G. D. Hair, Multiple-Constraint Adaptive Filtering. Dallas, TX: Texas Instrum. Sci. Services Div., 1969.

[14] O. L. Forst, III, "An algorithm for linearly constrained adaptive processing," Proc. IEEE, vol. 60, no. 8, pp. 926-935, Aug. 1972.

[15] K. Takao, H. Fujita, and T. Nishi, "An adaptive arrays under directional constraint," IEEE Trans. Antennas Propag., vol. AP-24, no. 5, pp. 662-669, Sep. 1976.

[16] A. M. Vural, "A comparative performance study of adaptive array processors," presented at the IEEE Int. Conf. Acoust., Speech Sig. Proc., May 1977.

[17] S. P. Applebaum and D. J. Chapman, "Adaptive arrays with main beam constraints," IEEE Trans. Antennas Propag., vol. AP-24, no. 5, pp. 650-662, Sep. 1976.

[18] M. H. Er and A. Cantoni, "Derivative constraints for broad-band element space antenna array processors," IEEE Trans. Acoust., Speech, Signal Process., vol. ASSP-31, no. 6, pp. 1378-1393, Dec. 1983.

[19] K. M. Buckley and L. J. Griffiths, "An adaptive generalized sidelobe canceler with derivative constraints," IEEE Trans. Antennas Propag., vol. AP-34, no. 3, pp. 311-319, Mar. 1986.

[20] C. Y. Tseng and L. J. Griffiths, "A unified approach to the design of linear constraints in minimum variance adaptive beamformers," IEEE Trans. Antennas Propag., vol. 40, no. 12, pp. 1533-1542, Dec. 1992.

[21] K. L. Bell, Y. Ephraim, and H. L. V. Trees, "A Bayesian approach to robust adaptive beamforming," IEEE Trans. Signal Process., vol. 48, no. 2, pp. 386-398, Feb. 2000

[22] F. Quian and B. D. Van Veen, "Quadratically constrained adaptive beamforming for coherent signal and interference," IEEE Trans. Signal Process., vol. 43, no. 8, pp. 1890-1900, Aug. 1995.

[23] B. D. Van Veen, "Minimum variance beamforming with soft response constraints," IEEE Trans. Signal Process., vol. 39, no. 9, pp. 1964-1972, Sep. 1991.

[24] D. D. Feldman and L. J. Griffiths, "A projection approach for robust adaptive beamforming," IEEE Trans. Signal Process., vol. 42, no. 4, pp. 867-876, Apr. 1994.

[25] L. C. Godara, "Application of antenna arrays to mobile communications, Part II: Beam-forming and direction-of-arrival considerations," Proc. IEEE, vol. 85, no. 8, pp. 1195-1245, Aug. 1997.

[26] H. Krim and M. Viberg, "Two decades of array signal processing research," IEEE Signal Process. Mag., vol. 13, no. 4, pp. 67-94, Jul. 1996.
[27] J. S. Reed, J. D. Mallett, and L. E. Brennan, "Rapid convergence rate in adaptive arrays," IEEE Trans. Aerosp. Electron. Syst., vol. AES-10, no. 6, pp. 853-863, Nov. 1974.

[28] D. H. Johnson and D. E. Dudgeon, Array Signal Processing: Concepts and Techniques. Englewood Cliffs, NJ: Prentice-Hall, 1993.

[29] H. L. Van Trees, Detection, Estimation, and Modulation Theory, Part IV, Optimum Array Processing. New York: Wiley, 2002.

[30] P. S. Naidu, Sensor Array Signal Processing. Boca Raton, FL: CRC, 2001.

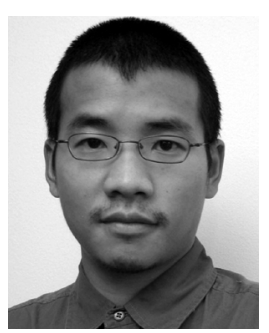

Chun-Yang Chen (S'05) was born in Taipei, Taiwan, R.O.C., on November 22, 1977. He received the B.S. and M.S. degrees in electrical engineering and communication engineering from National Taiwan University (NTU), Taipei, R.O.C., in 2000 and 2002, respectively, and is currently pursuing the $\mathrm{Ph} . \mathrm{D}$. degree in electrical engineering in the field of digital signal processing at the California Institute of Technology, Pasadena.

His interests include signal processing in MIMO communications, ultra-wideband communications,

and radar applications.

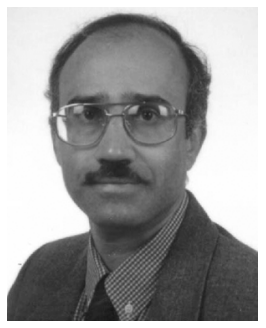

P. P. Vaidyanathan (S'80-M'83-SM'88-F'91) was born in Calcutta, India, on October 16, 1954. He received the B.Sc. (Hons.) degree in physics and the B.Tech. and M.Tech. degrees in radiophysics and electronics from the University of Calcutta, Calcutta, India, in 1974, 1977, and 1979, respectively, and the $\mathrm{Ph} . \mathrm{D}$. degree in electrical and computer engineering from the University of California at Santa Barbara in 1982.

He was a Postdoctoral Fellow at the University of California at Santa Barbara from 1982 to 1983 . In 1983, he joined the Electrical Engineering Department of the California Institute of Technology, Pasadena, as an Assistant Professor, and since 1993, he has been Professor of Electrical Engineering. His main research interests are digital signal processing, multirate systems, wavelet transforms, and signal processing for digital communications.

Dr. Vaidyanathan served as Vice-Chairman of the Technical Program committee for the 1983 IEEE International symposium on Circuits and Systems, and as the Technical Program Chairman for the 1992 IEEE International Symposium on Circuits and Systems. He was an Associate Editor for the IEEE TRANSACTIONS ON CIRCUITS AND SYSTEMS from 1985 to 1987, and is currently an Associate Editor for IEEE SignAL PROCESSING LETTERS, and a Consulting Editor for the journal Applied and Computational Harmonic Analysis. He has been a Guest Editor in 1998 for a special issues of the IEEE TRANSACTIONS ON SigNAL PROCESSING and the IEEE TRANSACTIONS ON CiRCUITS AND SySTEMS II, on the topics of filter banks, wavelets, and subband coders. He has authored a number of papers in IEEE journals, and is the author of the book Multirate Systems and Filter Banks. He has written several chapters for various signal processing handbooks. He was a recepient of the award for excellence in teaching at the California Institute of Technology for the years 1983-1984, 1992-1993, and 1993-1994. He also received the National Science Foundation's Presidential Young Investigator Award in 1986. In 1989, he received the IEEE ASSP Senior Award for his paper on multirate perfect-reconstruction filter banks. In 1990, he was recepient of the S. K. Mitra Memorial Award from the Institute of Electronics and Telecommuncations Engineers, India, for his joint paper in the IETE Journal. He was also the coauthor of a paper on linear-phase perfect reconstruction filter banks in the IEEE TRANSACTIONS ON SIGNAL PROCESSING, for which the first author (T. Nguyen) received the Young Outstanding Author Award in 1993. He received the 1995 F. E. Terman Award of the American Society for Engineering Education, sponsored by Hewlett Packard Co., for his contributions to engineering education, especially the book Multirate Systems and Filter Banks (Prentice-Hall, 1993). He has given several plenary talks including at the SAMPTA'01, EUSIPCO'98, SPCOM'95, and ASILOMAR'88 Conferences on signal processing. He has been chosen a Distinguished Lecturer for the IEEE Signal Processing Society for the year 1996-1997. In 1999, he was chosen to receive the IEEE Circuits and Systems Society's Golden Jubilee Medal. He is a recipient of the IEEE Signal Processing Society's Technical Achievement Award for 2002. 